\title{
Safety Evaluation of roller compacted concrete dam
}

\author{
Jing $\mathrm{Ma}^{1, \mathrm{a}}$, Lingqiang Yang ${ }^{2, \mathrm{~b}}$, Yan Wang ${ }^{2, \mathrm{c}}$ \\ ${ }^{1}$ School of Automation and Electrical Engineering, University of Jinan, Jinan, China 250022 \\ ${ }^{2}$ School of Civil engineer and architecture, University of Jinan, Jinan, Shandong province, China \\ acea_yanglq @ujn.edu.cn, cea_Gaor@ujn.edu.cn b, eo_wangy @ujn.edu.cn c
}

Keywords: Evaluation standard; roller compacted concrete dam; safety coefficient.

Abstract. The safety coefficient based on finite element method (FEM) has not corresponding evaluation standard, which limits the usage of FEM in roller compacted concrete dam (RCCD). In order to popularize FEM to RCCD more widely than ever, it is necessary to study the evaluation standard for RCCD safety coefficient based on FEM. In this paper, nonlinear FEM is used to calculate the safety coefficient of the RCCD. The strength reserve factor (SRF) method is adopted to simulate gradual failure and possible unstable modes of RCCD system. The entropy theory and catastrophe theory are used to obtain the ultimate bearing resistance and failure criterion of the RCCD. The most dangerous sliding plane for RCCD failure is found using the Latin hypercube sampling (LHS) and auxiliary analysis of partial least squares regression (PLSR). Finally a method for determining the evaluation standard of RCCD safety coefficient based on FEM is put forward using least squares support vector machines (LSSVM) and particle swarm optimization (PSO).

\section{Introduction}

Roller compacted concrete dams (RCCDs) are now constructed with great frequency in the world. Over the past two decades, many high RCCDs have been built, such as the 130m-high Baise dam and the 200.5m-high Guangzhao dam in China. Currently the highest dam of this type is Longtan Dam at 216m in China, with Diamer-Bhasha Dam planned at $272 \mathrm{~m}$ in Pakistan (Brian 2008).

The RCCD is mainly composed of the body and interface. The interface plays an important role as the weak point of the stability of the RCCD. The stability and safety of a RCCD to guard against sliding along the dam interface are key factors that must be addressed in the design stage of the RCCD. Generally speaking, analysis methods for the RCCD stability should include the rigid-body limiting equilibrium method (LEM), and finite element method (FEM). LEM is normally adopted before considering FEM. Ministry of Water Resources (SL-319-2005), et al. present evaluation standards for the LEM. Compared with the LEM, the FEM used in the calculation of anti-sliding analysis does not require any assumption of the sliding plane, but safety coefficient based on FEM has not corresponding evaluation standard, which limits the usage of FEM in RCCD. In order to popularize FEM to RCCD more widely than ever, it is necessary to study the evaluation standard for RCCD safety coefficient based on FEM.

\section{RCCD failure criterion}

RCC yield criterion

The transversely isotropic and elastoplastic model is used for the constitutive relationship of RCCD material. The isotropic and elastoplastic model is used for the constitutive relationship of the rock material of the dam foundation. The most typical Drucker-Prager (D-P) criterion (Drucker and Prager 1952; Zhou et al. 2008) in geotechnical engineering is used for the yield criterion of the RCCD and rock of the dam foundation:

$$
F=\alpha I_{1}+\sqrt{J_{2}}-K=0
$$

where I1 and $\mathrm{J} 2$ are the first invariant of stress tensor and the second invariant of deviatoric stress tensor, respectively. Both $\alpha$ and $\mathrm{K}$ are strength constants, which are related to cohesion $\mathrm{c}$ and internal 
friction angle $\varphi$. Different yield criterions can be realized in finite element calculations through changing the expressions of $\alpha$ and $\mathrm{K}$. In this paper, the D-P criterion of equivalent-area cones is used. Strength reserve factor method:

Zienkiewicz (Zienkiewicz and Pande 1977) firstly introduced strength reserve factor (SRF) method to elastoplastic FEM analysis. The dam and dam foundation have strength reserve ability. When shear strength parameters (cohesion $\mathrm{c}$ and internal friction coefficient $\mathrm{f}$ ) of the dam decrease, the dam will lose strength and become instability. Basic principle of SRF method is that the cohesion c and internal friction coefficient $\mathrm{f}$ are divided by the reduction coefficient $\mathrm{KR}$ :

$$
c_{R}=\frac{c}{K_{R}}, f_{R}=\frac{f}{K_{R}}
$$

With the gradual increasing of the reduction coefficient KR, the process of RCCD failure can be obtained, and the dam will reach a limit state.

\section{Determination of the most dangerous sliding plane of RCCD failure}

In this paper, the LHS method is adopted to generate the combinations of various mechanical parameters, and the elastoplastic finite element analysis is carried out based on the entropy catastrophe criterion, in the end, the most dangerous sliding plane is obtained by the sensitivity analysis of the mechanical parameters using the auxiliary analysis of PLSR.

Latin Hypercube Sampling

The LHS technique is a constrained sampling technique whereby the input parameter range is divided into equi-probable non-overlapping intervals. Let $\mathrm{N}$ denote the required number of realizations and $\mathrm{K}$ the number of random variables. The sampling space is then $\mathrm{K}$-dimensional. An $\mathrm{N} \times \mathrm{K}$ matrix $\mathrm{P}$, in which each of the $\mathrm{K}$ columns is a random permutation of $1, \mathrm{~L}, \mathrm{~N}$, and an $\mathrm{N} \times \mathrm{K}$ matrix $\mathrm{R}$ of independent random numbers from the uniform $(0,1)$ distribution are established. These matrices form the basic sampling plan, represented by the matrix $\mathrm{S}$ as

$$
S=\frac{P-R}{N}
$$

Each element of $\mathrm{S}$, sij, is then mapped according to its target marginal distribution as

$$
\hat{x}_{i j}=F_{x_{j}}^{-1}\left(s_{i j}\right)
$$

where $F_{x_{j}}^{-1}$ represents the inverse of the target cumulative distribution function for variable $\mathrm{j}$. A vector $\hat{X}_{i}=\left[\hat{x}_{i 1}, \hat{x}_{i 2}, \mathrm{~L}, \hat{x}_{i k}\right]$ now contains input data for one deterministic computation.

\section{Steps of determining the most dangerous sliding plane of the RCCD}

The steps of determining the most dangerous sliding plane of the RCCD are shown as follows:

(1) The deformation and strength parameters of the RCCD are taken as the effect factors, and the ranges of the factors are determined.

(2) The combinations of the effect factors are established by the LHS.

(3) The elastoplastic finite element model is established, and the safety coefficients of the dam failure corresponding to the combinations in step (2) are calculated using RCCD failure criterion.

(4) The sensitivities of mechanical parameters to the whole stability of the dam are determined using the auxiliary analysis of PLSR to analyze the combinations of the effect factors and corresponding safety coefficients of the dam failure. The interface of the most sensitive parameter is the most dangerous sliding plane.

\section{Evaluation standard analysis of RCCD safety coefficient}

Compared with conventional concrete dam, RCCD has obvious rolled characteristic and relatively weak construction interface of affecting dam stability. A lot of researches show that at the time of the 
RCCD failure, an interface is completely yield, and sliding channel is formed. Fig. 1 shows typical failure process of RCCD interface. Y-axis is strength reserve coefficient $\mathrm{Ks}$, X-axis is the interface yield rate PL.

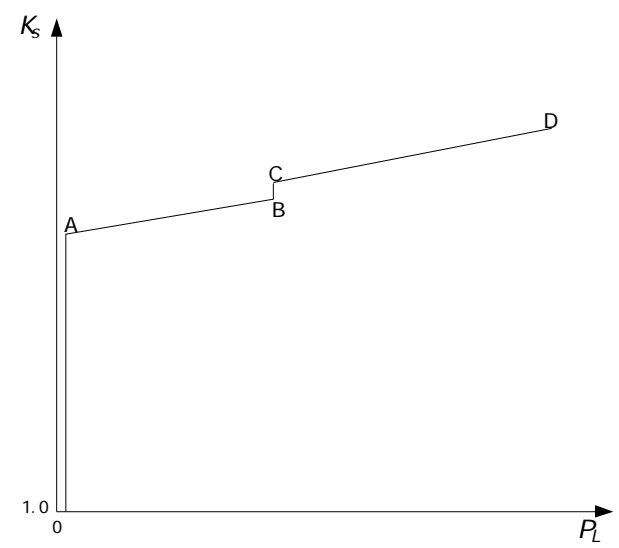

Fig.1 Typical failure process of RCCD interface

(1) Stability stage (before point A)

When $\mathrm{Ks}=1.0$, in addition to local small yield in the dam, the dam is basically in linear elastic state. With the increase of the Ks, yield area has a little expansion. Only when the Ks increases to certain value (corresponding point $\mathrm{A}$ ), the yield area begins to expand. The point $\mathrm{A}$ is called quasi-elastic state.

(2) Yield deformation stage (between point A and point C)

With the increase of the Ks from the point $\mathrm{A}$, the yield area increases rapidly to certain scope (about $30 \% \sim 50 \%$ ). Near the point B, the extension temporarily becomes slow until the point $\mathrm{C}$.

(3) Failure stage (between point $\mathrm{C}$ and point $\mathrm{D}$ )

The yield area expands rapidly from the point $C$. The upstream and downstream yield areas of the interface sharply joint together. Near the point $\mathrm{D}$, sliding channel is formed and whole sliding failure of the dam leads to the loss of the ability to dam operation.

\section{Determination of the evaluation standard for RCCD safety coefficient}

In the process of the RCCD failure, the quasi-elastic state is an important critical point. Before this point, the dam is mainly in elastic state and it is stable as a whole. After this point, the yield area rapidly expands until the dam failure. Therefore, the evaluation standard for RCCD safety coefficient based on the SRF method is given as:

$$
K_{e} \geq\left[K_{e}\right]
$$

where $\mathrm{Ke}$ is the safety coefficient in the quasi-elastic state, $[\mathrm{Ke}]$ is the allowable value of the Ke.

If the allowable value $[\mathrm{Ke}]=1.0$, the dam is very safe in the design condition. But in the process of dam operation, water level overload, work environment worsening, and the variation of mechanical parameters and other adverse factors will appear. Therefore, these factors must be considered into the impact of the [Ke]. Firstly, according to the results of the test and statistics, the ranges of the factors are obtained. Secondly, when the above factors change in the ranges at the same time, the [Ke] is obtained in the most adverse circumstance where the minimum of the quasi-elastic strength reserve coefficient appears. Specific expression is given as follows:

$$
\left[K_{e}\right]=1.0 \times\left(1+\left|K_{d}-K_{m}\right| / K_{d}\right)
$$

where $\mathrm{Kd}$ is the quasi-elastic strength reserve coefficient in the design condition, $\mathrm{Km}$ is the minimum of the quasi-elastic strength reserve coefficient with the variation of various factors.

\section{Example analysis}

The Longtan dam, the highest RCCD in the world currently, was built on the Hongshui River in China. Longtan RCCD was constructed in two phases. In the first phase normal water level is $375 \mathrm{~m}$, and the elevation of dam top is $382 \mathrm{~m}$. In the second phase normal water level is $400 \mathrm{~m}$, and the elevation of dam 
top is $406.5 \mathrm{~m}$. In this paper, 11\# dam section of right bank is selected in the first phase. The material partitions of Longtan 11\# dam section are shown in Fig. 2. According to the material partitions, RCC1, RCC2, RCC3 and foundation interfaces are looked as main interfaces. The FEM grid of Longtan 11\# dam section is shown in Fig. 3.

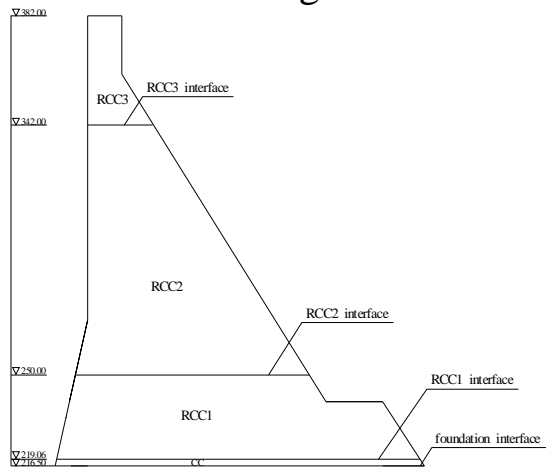

Fig. 2 Material partitions of Longtan 11\# dam section.

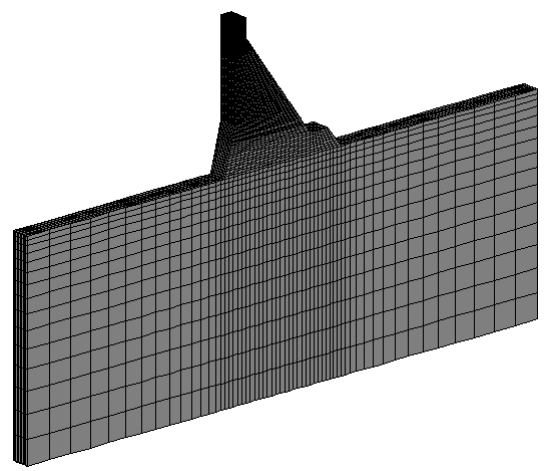

Fig. 3 The FEM grid of Longtan 11\# dam section

In this paper, RCC is regarded as transversely isotropic material. According to the material partitions shown in Fig. 2, there are eight groups of parameters that need to analyze. The horizontal and vertical elastic modulus of RCC1, RCC2 and RCC3 interfaces are Eli and Evi $(\mathrm{i}=1,2,3)$ respectively, the internal friction coefficient and cohesion of RCC1, RCC2 and RCC3 interfaces are fi and ci $(i=1,2,3)$ respectively, the horizontal and vertical elastic modulus of foundation interface are Elj and Evj ( $\mathrm{j}=4)$ respectively, and the internal friction coefficient and cohesion of foundation interface are $f j$ and $c j(j=4)$ respectively. According to the test result, the ranges of the mechanical parameters of the interfaces are shown in Table 1 and Table 2. The design values of main mechanical parameters are shown in Table 3 and Table 4, where Elk and Evk $(\mathrm{k}=5,6,7)$ are the horizontal and vertical elastic modulus of RCC1, RCC2 and RCC 3 bodies respectively, fk and $\mathrm{ck}(\mathrm{k}=5,6,7)$ are the internal friction coefficient and cohesion of RCC1, RCC2 and RCC3 bodies respectively, E8 and E9 are the elastic modulus of the conventional concrete and dam foundation rock respectively, f8 and $\mathrm{c} 8$ are the internal friction coefficient and cohesion of the conventional concrete and dam foundation rock respectively.

\section{Conclusions}

Evaluation standard for RCCD safety coefficient based on FEM is a problem requiring urgent solution. In this paper, the D-P criterion, SRF method, information entropy and catastrophe theory are applied to RCCD failure criterion. On this basis, the LHS and auxiliary analysis of PLSR are used to determine the most dangerous sliding plane of RCCD failure. Finally, LHS, LSSVM and PSO are adopted to establish evaluation standard for RCCD safety coefficient based on SRF method. The proposed method combines the advantages of several excellent algorithms, and can be popularized to any RCCD.

\section{Acknowledgements}

This work was financially supported by the Shandong Natural Science Foundation (ZR2014EL038).

\section{References}

[1] Olsson A., Sandberg G., and Dahlblom O. 2003. On Latin hyper cube sampling for structural reliability analysis. Structural Safety, 25, 47-68. 